Courant Mathematics and

Computing Laboratory

U.S. Department of Energy

\title{
Evaluation of Step Directions in Optimization Algorithms
}

William C. Davidon and Jorge Nocedal

Research and Development Report

Prepared under Contract DE-AC02-76ER03077

with the U.S. Department of Energy, Division of Basic

Energy Sciences, Applied Mathematical Sciences Program

Mathematics and Computers

December 1983 

Courant Mathematics and Computing Laboratory

New York University

EVALUATION OF STEP DIRECTIONS

IN OPTIMIZATION ALGORITHMS

William C. Davidon ${ }^{*}$ and Jorge Nocedal ${ }^{* *}$

December 1983

* Department of Mathematics, Haverford College, Haverford, PA 19041.

This work was done while this author was visiting the Courant

Institute of Mathematical Sciences

$* *$

Courant Institute of Mathematical Sciences, New York University, New York, NY 10012. Present address: EE/CS Dept., Northwestern University, Evanston, IL 60201.

Prepared under Contract DE-AC02-76ER03077

with the U. S. Department of Energy. 


\title{
DISCLAIMER
}

This report was prepared as an account of work sponsored by an agency of the United States Government. Neither the United States Government nor any agency thereof, nor any of their employees, makes any warranty, express or implied, or assumes any legal liability or responsibility for the accuracy, completeness, or usefulness of any information, apparatus, product, or process disclosed, or represents that its use would not infringe privately owned rights. Reference herein to any specific commercial product, process, or service by trade name, trademark, manufacturer, or otherwise, does not necessarily constitute or imply its endorsement, recommendation, or favoring by the United States Government or any agency thereof. The views and opinions of authors expressed herein do not necessarily state or reflect those of the United States Government or any agency thereof.

\author{
Printed in U.S.A.
}

Available from

National Technical Information Service

U. S. Department of Commerce

5285 Port Royal Road

Springfield, VA 22161 
TABLE OF CONTENTS

ABSTRACT

iv

1. Introduction I

2. Evaluation of a Step Direction 3

3. Application to Two Versions of the Conjugate

Gradient Algorithm $\quad 4$

4. Application to an Algorithm Based on Conic Functions 8

REFERENCES 


\begin{abstract}
We present a method for comparing that part of optimization methods which chooses each step direction. It is an example of a general approach to algorlthm evaluation in which one tests specific parts of the algorithm, rather than making overall evaluations on a set of standard test problems. Our testing procedure can be useful for developing new algorithms and for writing and evaluating optimization sof tware.
\end{abstract}

We use the method to compare two versions of the conjugate gradient algorlthm, and to compare these with an algorithm based on conic functions.

Key words and Phrases: optimization, sof tware evaluation, conic functions, conjugate gradients 


\section{Introduction}

A common procedure for comparing optimization algorithms consists of testing them on a standard set of problems. An evaluation is based on criteria such as the total number of function evaluations and the average number of successful runs; see for example Moré, Garbow and Hillstrom (1981). If these tests are carefully prepared they w1ll often glve a good Indication of the efficlency, rellablilty and robustness of optimization sof tware. Nevertheless, this overall evaluation sometimes does not provide enough detalled Information to help us determine the strengths and weaknesses of an algorithm. A program could perform poorly for very different reasons. For example, the line search could be Inadequate, in which case a simple modification could solve the difficulties; or the search directions could be poor, in which case there could be no cure. From these types of tests it may be very difficult to determine where the deflclencles are; see for example Hlebert (1981).

In this paper we present a method that testsea specific and very lmportant part of optimization algorlthms, namely the cholce of the step directions. For this we study a single iteration of each algorithm in an environment that emphasizes its essential character and minimizes the importance of programming decisions. The method is invariant under affine transformations of both the domaln and range of the objective function. It is used to compare two versions of the conjugate gradient algorlthm, and to compare these with an algorithm based on conic models. We study only algorithms that use line searches; however, we belleve that this approach could be useful for analyzing trust region methods as well.

The motivation for this work came from the development of an algorithm that uses conic models. There are many possible implementations of this algorithm, and some computational experience was desired. Overall evaluations on standard 
test problems could not be used, alnce there were too many unknowns. By using the evaluation method described in this paper, we have been able to observe the performance of the various conic models. We have also reexamined the conjugate gradient algorithm and found that the cholce of the two points used by the algorithm in generating a new search direction is important.

We believe that the major application of this approach will be in the development of new algorithms and the writing of their software. The efficiency of optimization codes is very dependent on various declsion parameters, such as the accuracy of the line search, the restart tolerance, or the trust region size. A good code is developed by choosing these parameters wisely, and its success depends largely on them. When a new type of algorithm is being developed, it is difficult to know how to choose the parameters; a great deal of numerical experimentation might be required. It would be preferable to find a way of isolating parts of the algorithm in order to gain useful information about each of the parameters. In this paper we present an example of how to do this. In Section 2 we outine a procedure for desiding how good a search direction is. We then show, in sections 3 and 4 , how one can vary some decision parameters independently and observe their effect on the search direction. We study here only the conjugate gradient method and a conic method. Other optimization algorithms require somewhat different procedures; however, the main idea remains the same.

By making a careful analysis of search directions Powell (1977) was able to explain why under some circumstances the Fletcher-Reeves method does not perform as well as the Polak-Ribiere method. The basis of his analysis is very similar in nature to the method presented here, and is evidence of the fruitfulness of this approach. 
Our goal is to develop a procedure for deciding how useful a direction is. The idea is to define an Inftial direction, let the algorlthm choose a new search direction, and by exploring the function alorig 1t, determine how good the new direction 1s. It is important that the procedure be invarient under affine transformations of both the domain and range of the objective function. We propose, among eeveral possible procedures, the following:

1. Choose an Initial point $x_{0}$ and an intial direction $d_{0}$.

2. Apply the algorithm and generate a new search direction $d_{i}$ (Call $x_{i}$ the point where $d_{0}$ and $d_{1}$ intersect, $1 . e .$, the starting point of the new Iteration).

3. Locate the minimizer $x_{\star}$ of the function along $d_{i}$. Compute the relative decrease in $\mathrm{f}$ :

$$
I=\frac{f_{1}-f^{\star}}{f_{0}-f_{1}},
$$

where $f_{\star}, f_{0}$ and $f_{1}$ are the function values at $x_{\star}, x_{0}$ and $x_{1}$.

The quotient $r$ will be the measure of the quality of a search direction. We assume that, in the region of interest, there is a unique one-dimensional minimizer of the function along $d_{1}$. Note that some algorithms may require additional information in step 2. For example, variable metrlc methods require a matrix that is updated during the iteration.

To apply this procedure we start by choosing a test function, for example one with singularlties or narrow curving valleys. Next we select the starting point and direction in a region of interest of the function. We then apply the varlous algorithms to be tested and record how good their search directions are. We will now describe in detall two applications of this procedure. 
3. Application to two versions of the conjugate gradient algorithm

The confugate gradient algorlthm generates a segrch difection based on Information about the function at two points; see for example Fletcher and Reeves (1964). It is common to use for this the inftial and final points in the line search. As the starting polnt is fixed, the only varlable in the algorlthm is the second point. In other words, the only free parameter of the algorithm is the accuracy in the line search. We will not follow this practice, but will allow more freedon in choosing the two points required by the algorlthm. During the line search, aeveral. intermediate points are computed and some of these can be considered as candidates.

To study the effect of the cholce of the two points on the search directions we will consider two strategies:

$\operatorname{CG}(1)$ We use the starting point, which is given, and the one-dimensionel minimizer of the function along the initial direction.

CC(2) We also find the one-dimensional minimizer, which will be one of the points used. The second point is either the starting polnt or the first trial point in the Ifne search, whlchever gives the lowest function value. The cholce of the trial point will be arbitrary.

We will now comment on these strategles. The first one resembles the common use of the conjugate gradient algorithm, except for the use of an accurate one-dinensional minimizer. This can be changed to find only a rough estimate of the minimizer, but this introduces some complications, as will be discussed later.

The second strategy is based on the observation that even though the conjugate gradient algorithm requires only two data points to generate a new search direction, it should use at lesst three points during the line search. This is because we want to ensure that the line search performs at least one 
quadratic fit, so that if the objective function is quadiatic the algorithm w11 maintain conjugacy. Therefore for every search direction the method will generate at least three points: the starting point, the trial point and an estimate of the minimizer. It may generate other intermediate points depending on the objective function, the type of line search, and the accuracy of the search. It is not desirable to consider the intermediate points, because they depend on the detalls of the line search. We therefore opted for considering only three candidate points: the starting point and the minimizer (which are uniquely specified) and the trial point which will be considered as the variable in our tests. The reason that the trial step is not fixed is that there is no accepted way of choosing it. Various suggestlons have been implemented in the modern codes, but they are to some extent arbitrary. In the numerical tests described below we sill vary the value of the trial point within a prescribed interval around the minimizer and will observe its effect in the generation of search directions.

The following results were obtalned using double precision arithmetic on a VAX $11 / 780$ at the Courant Mathematics and Computing Laboratory. The starting point is denoted by $x_{0}$, the initial direction by $d_{0}$ and the minimum of the function along $d_{0}$ by $f_{1}$. The steplength to the minimizer along the initial direction is denoted by $\lambda$, and the minimum value of the function along the new search direction by $f_{\star}$. The relative decrease in the function, denoted by $r$, is given by (1). The test functions are taken from More', Garbow and Hillstrom $(1981)$

Problem 1 Rosenbrock's function, $x_{0}=(0,0)^{t}, d_{0}=(1,0)^{t}$

$$
\lambda=.161, \mathrm{f}_{1}=.771, \mathrm{f}_{0}=1.00
$$

For $\operatorname{co}(1): f_{\star}=.624, r=.642$ 
For $\operatorname{CG}(2)$ :

TRIAL STEPLENGTH

\begin{tabular}{|l|llllll|}
\hline \multirow{2}{*}{$\mathrm{f}$} & .01 & .05 & .167 & .3 & .5 & .1 \\
\cline { 2 - 7 } $\mathrm{f}$ & .626 & .615 & .474 & .624 & .624 & .624 \\
& .633 & .681 & 1.30 & .642 & .642 & .642 \\
\hline
\end{tabular}

Problem 2 Rosenbrock's function, $x_{0}=(0,0)^{t}, d_{0}=(.1,-.015)^{t}$

$$
\lambda=.102, \mathrm{f}_{1}=.872, \mathrm{f}_{0}=1.00
$$

For $\operatorname{cc}(1): \mathrm{f}_{\star}=.729, \mathbf{r}=1.12$

For $\operatorname{CG}(2)$ :

TRIAL STEPLENGTH

\begin{tabular}{|l|cccccc|}
\hline \multirow{2}{*}{$\mathrm{f \star}$} & .01 & .05 & .1 & .2 & .6 & 2.0 \\
\cline { 2 - 7 } & .719 & .670 & .588 & .729 & .729 & .729 \\
& 1.19 & 1.58 & 2.22 & 1.12 & 1.12 & 1.12 \\
\hline
\end{tabular}

Problem' 3 Powell's singular function, $x_{0}=(2,-.9, .2,-.2)^{t}$

$$
\mathrm{d}_{0}=(-1.7, .8,-.1, .7)^{\mathrm{t}}, \lambda=2.08, \mathrm{f}_{1}=1.21, \mathrm{f}_{0}=287
$$

For $\operatorname{CG}(1) \mathrm{f}_{\star}=.819, \mathrm{r}=.0014$

For $\operatorname{CG}(2)$ :

TRIAL STEPLENGTH

\begin{tabular}{|l|cccccc|}
\hline \multirow{2}{*}{$\approx$} & 1.0 & 1.5 & 2.0 & 3.0 & 6.0 & 15. \\
\cline { 2 - 7 } & .630 & 1.99 & .255 & .783 & .819 & .819 \\
& .0020 & .0035 & .0033 & .0015 & .0014 & .0014 \\
\hline
\end{tabular}

We can see from these results that the second strategy, $\operatorname{co}(2)$, is usually better. When the trial point is near the one-dimensional minluizer, $C G(2)$ 
generates a much better search direction than CG( 1$)$.

We present only a small sample of results, but it is representative of what we have observed. The purpose of this paper is to describe an approach for evaluating algorithms; therefore we will not make extensive comparisons of different methods. Note that there is very little extra computational cost in $\operatorname{CG}(2)$ and that this type of strategy is not difficult to implement. Therefore we suggest that it should be tried in the varlous confugate gradient-type codes currently in use. There are several precautions one should take while dolng this. The two points may be placed so that large numerical errors are Introduced while computing the new search direction. One should take this into account before deciding which two points to use. Also, the condition sty>0 should be enforced if the algorithm performs a variable metric update (here $s$ represents the difference between the two points used and $y$ the difference in gradients). Examples of these methods are the code of Shanno and Phua (1980) and variable storage methods.

We should point out that, even though in the results presented above tha second 'strategy is always preferable. there are cases where it is not. However this occurs rarely and in the majorlty of the tests $\operatorname{co}(2)$ is superior.

The evaluation method just described makes use of accurate one-dimensional minimizers. This simplifies the testing but does not reflect the practical use of optimization algorithms. This can be changed and rough estimates of the minimizer can be used. However, one should make sure that the cholce of this estimate of the minimizer is the same for all algorithms tested, and if possible, that this cholce is invariant under affine transformations of both the domain and range of the function.

Overall our testing procedure suggests that the selection of the two points can be Important in the confugate gradient method, and that the common practice of performing the simplest possible line search may not be justified. 
4. Application to an algorithm based on conic functions

We will now consider our testing procedure to evaluate a new optimization algorithm, that will be called the conic algorithm. It is an extension to general functions of the methods described by Davidon(1982) and Gourgeon and Nocedal(1982). To generate a new search direction the conlc algorithm requires function information at three points. These must satisfy certain conditions to ensure the existence of a normal conic fit. In the tests described below the three points are the starting point, the one-dimensional minimizer and the first trial point in the line search. As in the case of the confugate gradient method the trial point will be allowed to vary, but must be such that a normal conic fit is possible.

We will choose an initial point and direction, and will study the effect of the trial point on the new search direction. This algorithm will be compared with CG(2), the better of the two conjugate gradient methods. The conic algorithm used in these tests has approximately the same storage and computational requirements as the conjugate gradient method; therefore we are comparing similar algorithms.

Here are some results on the same functions tested in the previous section. The problem numbers correspond to those of section 3. 
Problem 1

\begin{tabular}{|l|l|llllll|}
\hline \multicolumn{2}{|c|}{ TRIAL STEPLENGTH } & .01 & .05 & .167 & .3 & .5 & 1.0 \\
\hline \multirow{3}{*}{ CONIC } & $\mathrm{f}^{\star}$ & .546 & .487 & .340 & .332 & .447 & .556 \\
\cline { 2 - 8 } & $\mathrm{r}$ & .983 & 1.24 & 1.88 & 1.92 & 1.41 & .939 \\
\hline \multirow{3}{*}{$\operatorname{cG}(2)$} & $\mathrm{f}^{\star}$ & .626 & .615 & .474 & .624 & .624 & .624 \\
\cline { 2 - 8 } & $\mathrm{r}$ & .633 & .681 & 1.30 & .642 & .642 & .642 \\
\hline
\end{tabular}

Problem 2

\begin{tabular}{|l|c|cccccc|}
\hline \multicolumn{2}{|c|}{ TRIAL STEPLENGTH } & .01 & .05 & .1 & .2 & .6 & 2.0 \\
\hline \multirow{3}{*}{$\operatorname{CONIC}$} & $\mathrm{f}^{\star}$ & .466 & .499 & .452 & .504 & .654 & .710 \\
\cline { 2 - 8 } & $\mathrm{r}$ & 3.17 & 3.30 & 3.28 & 2.87 & 1.70 & 1.27 \\
\hline \multirow{2}{*}{$\operatorname{CG}(2)$} & $\mathrm{f}^{\star}$ & .719 & .670 & .589 & .729 & .729 & .729 \\
\cline { 2 - 8 } & $\mathrm{I}$ & 1.19 & 1.58 & 2.22 & 1.12 & 1.12 & 1.12 \\
\hline
\end{tabular}

Problem 3

\begin{tabular}{|c|c|c|c|c|c|c|c|}
\hline \multicolumn{2}{|c|}{ TRIAL STEPLENGTH } & .1 & .5 & 1.0 & 1.5 & 2.0 & 3.0 \\
\hline \multirow{2}{*}{ CONIC } & $f *$ & .678 & .606 & .377 & .251 & .261 & .790 \\
\hline & $\mathbf{r}$ & .0019 & .0021 & .0030 & .0033 & .0033 & .00147 \\
\hline \multirow{2}{*}{$\cos (2)$} & $f^{\star}$ & .813 & .774 & .630 & .199 & .255 & .783 \\
\hline & $\mathbf{r}$ & .0014 & .0015 & .0020 & .0035 & .0033 & .0015 \\
\hline
\end{tabular}


In these tests the conic algorithm compares favorable with the best strategy for the conjugate gradient method, and is definftely more successful than the usual confugate gradient method CG(1). Again, we oniy present a small set of results and leave detalled comparisons for a future study.

The main conclusion drawn from these tests is that conic fits are much more stable than anticipated. The practical implementation of confc methods has been slowed down by the lack of clear criterla for choosing three points that wil generate a good conlc models. It was feared that different cholces could glve drastically different results, because conlc functions vary rapidiy in magnitude near a singular hyperplane. The tests have shown that as long as the three polnts are not too widely separated, the conic models will produce good estimates of the minimizers. This can be done by safeguarding the line search using one of the usual controls. Note that the confc algorithm is not much more sensitive than the conjugate gradient method to the cholce of the trial steplength. Several other aspects remaln to be studied before conlc algorlthms can be implemented in general purpose codes. The evaluation procedure described in this paper will be useful for these studies. Up to now 1 t suggests that conic models can make a contribution in the development of algorithms for nonlinear optimization. 


\section{References}

1. Davidon, W.C. (1982). Confugate directions for conic functions, In M.J.D. Powe 11, ed., Nonlinear Optimization 1981, Academic Press.

2. Fletcher, R. and Reeves, C. (1964). Function minimization by conjugate gradients, Computer Journal $7,149-154$.

3. Gourgeon, H. and Nocedal, J. (1982). A conic algorithm for optimization, Tech. Rep. Courant Institute, New York University, to appear in SIAM J. Scientific Stat. Computing.

4. Hiebert, K. L. (1981). An evaluation of mathematical software that solves nonlinear least squares problems, ACM Trans. Math. Sof tware 7, 1-16.

5. Moré, J. J., Garbow, B. S. and Hillstrom, K. E. (1981). Test1ng unconstralned optimization sof tware, ACM Trans. Math. Software 7, 17-41.

6. Powell, M.J.D. (1977). Restart procedures for the conjugate gradient method, Math. Programming 12, 241-254.

7. Shanno, D, and Phua, K. (1980). Remark on Algorithm 500, ACM Trans. Math. Sof tware 6,4. pp. 618-622. 
This book may be kept

FEB. OS 1984

FOURTEEN DAYS

A fine will be charged for each day the hook is kept overtine.

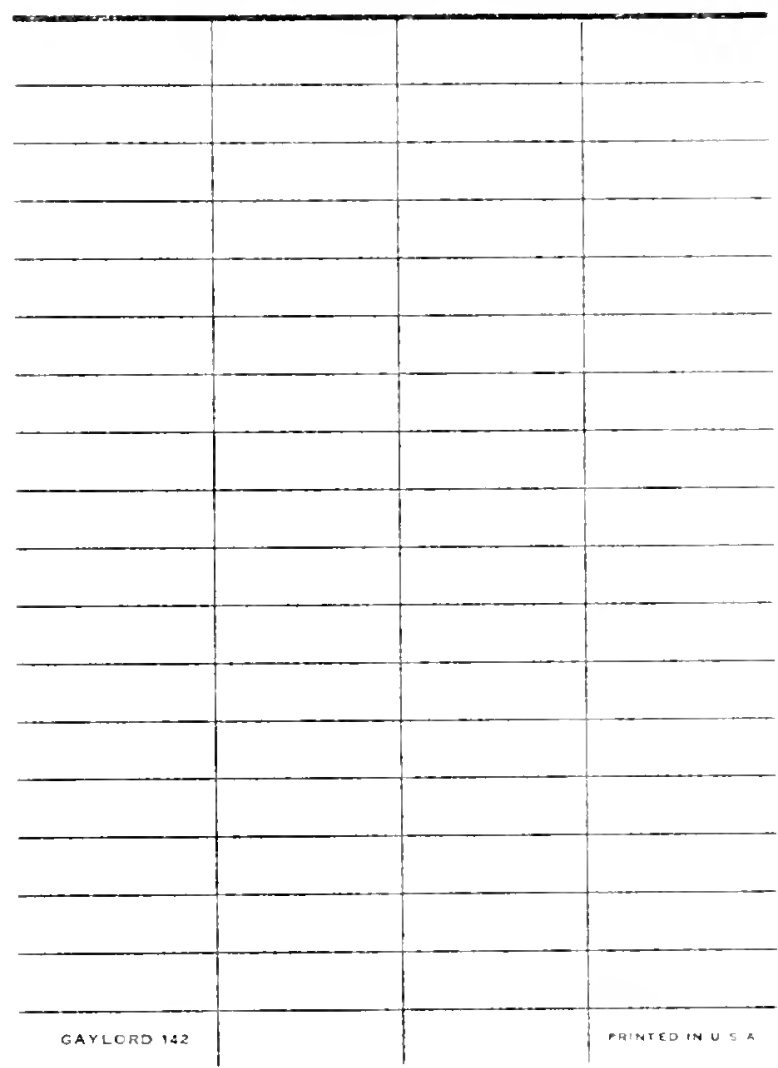


03077-206 Davidon

Evaluation of step directions

in optimization algorithms.

\section{LIBRARY}

N.Y.U. Courant Institute of

Mathematical Sciences

251 Mercer St.

New York, N. Y. 10012 
